\title{
Rheological behaviours of silica/water, silica/PEG systems and mechanical properties of shear thickening fluid impregnated Kevlar composites
}

\author{
EBRU SARALOĞLU GÜLER \\ Faculty of Engineering, Baskent University, Bağlıca Kampüsü, Fatih Sultan Mahallesi Eskişehir Yolu, 06790 \\ Etimesgut/Ankara, Turkey \\ esguler@baskent.edu.tr
}

MS received 2 October 2017; accepted 23 December 2017; published online 30 July 2018

\begin{abstract}
Evolution in armour materials is critical to improve the protection abilities together with the properties of flexibility and lightness. Silica colloidal suspensions are important candidates to improve strength of the armour materials. The dispersing medium of the silica particles acts as an important role in the formation of shear thickening fluids (STF). STF has the ability to have increased viscosity by increasing the shear rate. Rheological, impact, flexibility and yarn pull-out tests were performed during the study. This study demonstrated that STF did not occur in the case of dispersing silica in water, but increased the impact resistance when Kevlar treated by the silica dispersed in water as compared to neat Kevlar. Fumed silica distribution in polyethylene glycol (PEG) led to STF creation confirmed by the rheological tests. The impact resistance of STF-treated Kevlar was found to be improved when compared to silica-dispersed water-impregnated Kevlar. It was observed that the pull-out force values of STF-fertilized Kevlar were increased compared to neat Kevlar.
\end{abstract}

Keywords. Shear thickening fluid; silica; Kevlar; polyethylene glycol; impact; yarn pull-out.

\section{Introduction}

Body armour materials are evolving to sustain protection against stabbing and ballistic threats. Armours are classified as hard and soft body armours that are used during high risk threats and every day life of police officers and security guards, respectively [1]. Hard armours lack heavy and bulky and restrict the mobility, so that the flexibility and weight are important issues that were to be considered. Ceramic plates, steel plates with fabric, fibre-reinforced polymers, light alloys are used as armour materials now. However, currently used body armours are still too heavy with limited flexibility.

As it is known, the viscosity can either increase or decrease by increasing the shear rates called shear thickening and shear thinning, respectively, in non-Newtonian fluids [2]. For instance, $61 \mathrm{vol} \%$ (72.5 wt $\%$ ) $\mathrm{SiO}_{2}$ and $54 \mathrm{vol} \%$ (62 wt \%) CS samples show shear thickening behaviour, whereas shear thinning response was observed in $48 \% \mathrm{SiC}$ that seems to be plateau to a Newtonian behaviour at high strain rates [3]. The viscous relation was explained by a common model, i.e., 'power low model' $\left(\eta=m \dot{\gamma}^{n-1}\right)$, where $\eta, m, n$ and $\dot{\gamma}$ are viscosity, function of temperature, power low index and shear rate, respectively [2,4]. Most of the non-Newtonian materials exhibit shear thinning, $n<1$, whereas shear thickening regions are observed in some of the concentrated suspensions, $n>1[2,4]$. In addition, it was claimed that the model is valid for the region where viscosity is increased by shear rate, $n>1$ [4-6]. Shear thickening is specified as the increase in the viscosity upon increase in the applied shear rate and defined as the increase in volume upon deformation $[7,8]$. Shear thickening fluid (STF) is type of non-Newtonian fluid that has the ability of possessing increased viscosity by increasing shear rate $[9,10]$. STFs that have a colloidal nature are composed of dispersed and suspended solid particles in fluids [11]. A striking increase in the viscosity of STF occurs at a critical shear rate $[9,12]$. Furthermore, as the silica concentration in PEG is increased from 65 to $70 \mathrm{wt} \%$, the beginning point of the increase in viscosity by shear rate was decreased [13]. Moreover, viscosity is increased with an increase in the particle concentration due to easier formation of hydro-clusters that led to shear thickening at lower shear rates [14]. The striking point is increase in the viscosity, i.e., shear thickening corresponds to the beginning of the overwhelming of hydrodynamic interaction $[15,16]$. The particles are separated by the repulsive inter-particle forces below the critical point, leading to a relatively low viscosity and ordered structure [15]. Hydrodynamic forces overwhelm the repulsive inter-particle (electrostatic) forces that accelerates the aggregation and destruction of the order of the suspended particles [15-17]. The interaction of the dispersed particles and the fluid medium is explained by three forces that are hydrodynamic, inter-particle and Brownian forces [18]. Shear thickening behaviour is displayed as a result of 'silica hydro-cluster' development by hydrodynamic forces [17]. The clusters of 
silica particles that cannot be disturbed by shear are showed in the study of Raghavan and Khan [7]. Moreover, lower particle sizes provide great convenience to agglomeration due to the increased surface area and surface hyrdroxyl groups that are capable of making hydrogen bonding [12]. The source of the Brownian forces depending on the Brownian diffusion is the tendency of particles to move towards regions of lower concentration, since the particles interfere with others where the particle concentration is high [2]. Therefore, there is a direct contribution of Brownian forces between particles in the viscosity when there is an anisotropic distribution of nearby particles according to Mewis and Wagner [2].

The colloidal silica is generally composed of dispersing the particles in a solvent (medium) like water [19], ethylene glycol [14,20,21], PEG [7,22-26], PE [27] and polystyrene [27]. $\mathrm{SiC}, \mathrm{SiO}_{2}, \mathrm{TiO}_{2}$, corn-starch (CS) and their mixtures are among the types of dispersed solid particles [3,27]. STF formation was achieved by dispersing the particles of $\mathrm{SiO}_{2}$, $\mathrm{CS}$ and mixture of $\mathrm{SiO}_{2}$ and $\mathrm{SiC}$ in ethylene glycol according to the study of Petel et al [3]. The general procedure for the impregnation of STF to woven fabrics involves placing woven fibres in STF for 1 min followed by the dilution of STF by ethanol $[25,28]$. Then, the specimens were settled in oven for drying at $80^{\circ} \mathrm{C}$ for $20 \mathrm{~min}$ [28]. Wetzel et al [29] claimed that the rheological behaviours of STF samples are not altered by ethanol removal. On the other hand, ethanol addition led to increase in the critical shear rate and decrease in final viscosity of $40 \mathrm{wt} \%$ silica nanoparticle dispersed in the polymer $[25,28]$. Sonochemical mixing is also recommended before evaporation to obtain improved shear thickening effect [10]. The surface treatment of silica particles before dispersing in the PEG resulted in improved shear thickening behaviour with an increase in particle concentration [12].

The effect of temperature $\left(-40\right.$ to $\left.40^{\circ} \mathrm{C}\right)$ on viscosityshear rate behaviour was studied in silica-dispersed PEG and polypropylene glycerol [26]. It was concluded that decreasing temperature increased the viscosity and decreased the critical shear rate $[16,17,26,30]$. The reason for decreasing effect of temperature rise on the viscosity can be explained by the development of the Brownian motion of the particles and the decrease in the strength of 'solvent-silica hydrogen bond' at higher temperatures resulted in the enhancement of disordered structure [17]. Mewis and Biebaut [31] agreed with the decreasing effect of diminishing temperature on the critical shear rate, but they claimed that viscosity decreases in the shear thinning range, since decreasing temperature leads to shrinkage of the polymer coat (stabilizer layer). Moreover, increasing temperature led to decrease in the shear thickening region [31]. Because cage coherency, which is proportional with crowded particles was decreased when temperature was increased from 20 to $60^{\circ} \mathrm{C}$ for all particle volume fractions [32]. The rise in the temperature may affect cluster formation adversely and lead to decrease in the viscosity. Moreover, raising temperature led to softening of the particles that results in disappearance of the shear thickening [8]. It was also suggested that the temperature effect $\left(20-35^{\circ} \mathrm{C}\right)$ is more pronounced in more concentrated systems [31]. Furthermore, shear thickening behaviour was observed in the dispersion of $2.6 \%$ starch at $60^{\circ} \mathrm{C}$ only if it was heated for $15 \mathrm{~min}$ [33]. On the other hand, shear thinning was seen when the times were $30,90,210$ and $540 \mathrm{~min}$ at the same temperature, because granules were softened by the exposed time at $60^{\circ} \mathrm{C}$ [33]. Furthermore, particle softening was observed as the decrease in viscosity by increasing shear rate at all stages that are 15 , 30 and $45 \mathrm{~min}$ at $65^{\circ} \mathrm{C}$ during $25 \%$ aqueous starch dispersion [8]. Another point of view on the temperature effect is the improvement of polymer (PEG) adsorption on silica surface when the temperature was increased from 15 to $35^{\circ} \mathrm{C}$ due to the development of polymer coils [34].

The impact resistance is studied by Decker et al [35], which claimed that STF composed of silica (52 vol\%) dispersed in PEG, damaged less compared to neat Kevlar when $2.33 \mathrm{~kg}$ load is dropped from $0.5 \mathrm{~m}$. Moreover, the ballistic properties of the STF-treated Kevlar fabrics were studied, where silica colloidal water suspension of different concentrations in water was used as STF [19]. On the other hand, aqueous suspension of silica particles with $40 \mathrm{wt} \%$ was used and the medium was changed to ethylene glycol followed by drying, in the study of Lee et al [21]. STF (silica/PEG)impregnated glass fabric also revealed better results compared to neat glass fabrics during quasi-static stab-resistant tests for knife threats [22]. Computational model and numerical simulation were developed for prediction of ballistic responses for the STF-impregnated fabric and confirmed by the ballistic tests $[36,37]$. The compression tests were conducted for silica/PEG-impregnated warp-knitted spacer fabrics and it was concluded that the more STF impregnation resulted in better damping behaviours [38].

Yarn pull-out tests express the friction between the yarns in a woven fabric [1]. Yarn pull-out tests were conducted by clamping the fabric by two grips placed at the left and right hand sides $[39,40]$. On the other hand, the fabric specimen was clamped by flat-faced upper grid and V-grooved lower grip [19] or flat grip providing the single yarn to be cut [41]. All the setups include the yarn that is going to be pulled out was let to stay in the middle of the specimen $[13,39,40]$. The yarn pullout force or the peak force that is necessary to remove one of the yarns from the other is critical to understand the friction between the yarns in the fabrics [1]. It was concluded that the single yarn pull-out force depends on the fabric length; if the fabric length is from 50 to $350 \mathrm{~mm}$, the resultant force is increased from $\sim 14$ to $70 \mathrm{~N}$ [42]. In addition, it is claimed that multiple yarn pull-out force is greater than the single yarn pull-out force [42].

In this study, the effects of two different silica dispersion mediums, water and PEG on the formation of STF, impact properties of colloidal silica-treated Kevlar specimens were studied. Furthermore, the flexibilities of neat Kevlar, 30 and $40 \mathrm{wt} \%$ fumed silica dispersed in PEG were compared. Single yarn pull-out tests were applied to neat Kevlar and STF composed of $40 \mathrm{wt} \%$ fumed silica and PEG-treated Kevlar specimens at two different pull out rates. 
Table 1. Impact test parameters and samples.

\begin{tabular}{|c|c|c|c|c|c|c|c|}
\hline $\begin{array}{l}\text { Sample } \\
\text { no. }\end{array}$ & $\% \mathrm{SiO}_{2}$ & $\begin{array}{l}\mathrm{SiO}_{2} \text { dispers- } \\
\text { ing medium }\end{array}$ & $\begin{array}{l}\text { Ply number } \\
\text { (layer) }\end{array}$ & $\begin{array}{l}\text { Drop mass } \\
(\mathrm{kg})\end{array}$ & $\begin{array}{l}\text { Drop } \\
\text { height } \\
\text { (m) }\end{array}$ & $\begin{array}{l}\text { Impact } \\
\text { velocity } \\
\left(\mathrm{m} \mathrm{s}^{-1}\right)\end{array}$ & $\begin{array}{l}\text { Impact } \\
\text { energy }(J)\end{array}$ \\
\hline 1 & 0 (neat Kevlar) & - & 1 & 11.5 & 0.2 & 1.98 & 22.54 \\
\hline 2 & 40 & Water & 1 & 11.5 & 0.2 & 1.98 & 22.54 \\
\hline 3 & 40 & Water & 1 & 11.5 & 0.48 & 3.07 & 54.10 \\
\hline 4 & 70 & Water & 1 & 11.5 & 0.2 & 1.98 & 22.54 \\
\hline 5 & 70 & Water & 1 & 11.5 & 0.48 & 3.07 & 54.10 \\
\hline 6 & 40 & PEG & 3 & 11.5 & 1.77 & 5.90 & 200 \\
\hline 7 & 40 & PEG & 5 & 11.5 & 1.77 & 5.90 & 200 \\
\hline
\end{tabular}

\section{Materials and methods}

\subsection{Materials}

Silica of $22 \mathrm{~nm}$ size suspended in water with an amount of $40 \mathrm{wt} \%$, fumed silica (AerosilD200-specific surface area of $200 \mathrm{~m}^{2} \mathrm{~g}^{-1}$ ) and polyethylene glycol (Sigma AldrichMW 200) were used during the study. The Kevlar fabric used during composite production was plain-woven with an areal density of $200 \mathrm{~g} \mathrm{~m}^{-2}$. All tested fabric specimens were $110 \mathrm{~mm} \times 110 \mathrm{~mm}$.

\subsection{STF preparation}

STF samples were prepared by mixing proper amounts of ingredients by mechanical stirrer at $1000 \mathrm{rpm}$ for $2.5 \mathrm{~h}$. All the as-prepared samples were then ultrasonicated at $50^{\circ} \mathrm{C}$ for 20 min by SonorexRK255 H Ultrasonic Bath.

The $40 \mathrm{wt} \%$ colloidal silica, which is dispersed in water was directly used for samples 2 and 3 (table 1). The silica concentration was increased to $70 \%$ by evaporating the water. Furthermore, the colloid was heated at $120^{\circ} \mathrm{C}$ for $60 \mathrm{~min}$ to produce STFs for samples 4 and 5 (table 1).

Fumed silica was dispersed in PEG with amounts of 30 and $40 \mathrm{wt} \%$ for the preparation of STF used in samples 6 and 7. PEG was chosen as solvent due to its properties including non-toxicity, low volatility and thermal stability [38].

\subsection{Rheological test}

The STF specimens were subjected to rheological tests using Anton-Paar MCR301 at room temperature before dilution by adding ethanol.

\subsection{Impregnation}

Plain-woven Kevlar with an areal density of $200 \mathrm{~g} \mathrm{~m}^{-2}$ were used. STF samples were impregnated on square fabric specimens for 20 min by rolling using a steel cylinder followed by the addition of excess amount of ethanol. Then, the impregnated specimens were heated at $120^{\circ} \mathrm{C}$ for $1 \mathrm{~h}$.

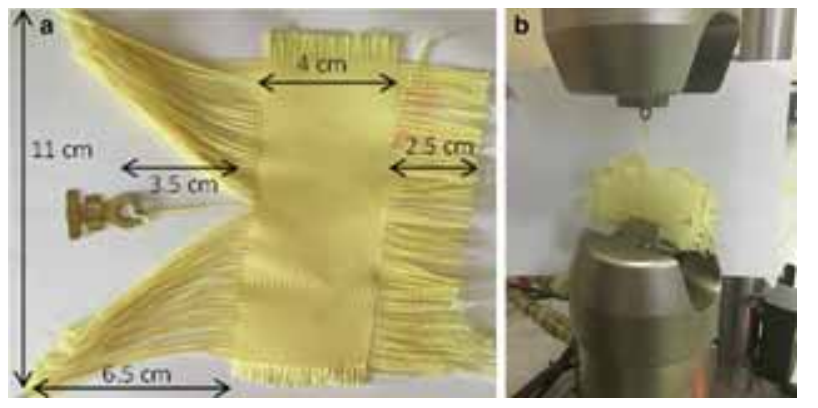

Figure 1. (a) The frayed regions and the test length $(4 \mathrm{~cm})$ of the fabric sample. (b) Yarn pull-out test setup.

\subsection{Impact and flexibility test}

Impact tests were conducted by dropping $11.5 \mathrm{~kg}$ load from various heights to observe the failure behaviour of the STFimpregnated and neat Kevlar fabrics. The details of the samples and applied test conditions are given in table 1. Sample no. 1 is neat Kevlar, in other words, the sample before impregnation. Comparison of samples 2 and 3, 4 and 5 can be done regarding the drop height. In addition, samples 2 and 4, 3 and 5 can be compared to observe the effect of silica content. Samples 6 and 7 were prepared by $40 \mathrm{wt} \%$ silica because of the improved rheological properties.

The flexibilities of neat Kevlar, 30 and $40 \mathrm{wt} \%$ fumed silica dispersed in PEG were tested by using $11.5 \mathrm{~kg}$ mass. The samples were fixed from two sides and the weight was left on the samples.

\subsection{Yarn pull-out test}

Yarn pull-out tests were conducted by using STF specimens composed of $40 \mathrm{wt} \%$ fumed silica and PEG due to improved rheological results. Figure 1a and b displays the dimensions of the fabric sample and yarn pull-out test setup, respectively. The lower part of the fabric was clamped in the base of Instron 8874 tensile test machine. However, the lower end of the single yarn that was going to be pulled out was carefully excluded 
and was not fastened in the lower jaw to let it leave. The single yarn was tied up to an attachment that clamped in the top jaw of the machine. The pull out rate was kept at $60 \mathrm{~mm} \mathrm{~min}^{-1}$ for the neat Kevlar since the test behaviour is not affected by the change in the pull out rate [41]. The pull out rates of both 60 and $70 \mathrm{~mm} \mathrm{~min}^{-1}$ were studied for the STF specimens composed of $40 \mathrm{wt} \%$ fumed silica and PEG.

\section{Results and discussion}

\subsection{STF rheological properties}

The steady shear viscosity as a function of steady shear rate is shown in figure 2 for silica at amounts of 60 and $70 \mathrm{wt} \%$ dispersed in water. Shear thinning response was observed, i.e., severe contrast to shear thickening behaviour [6]. These samples can be considered as shear thinning fluids in the range of measured shear rates [3]. In addition, $80 \mathrm{wt} \%$ of silica dispersed in water was tried, but the sample cannot be tested adequately due to its increased density. The reason for choosing water as the dispersing medium is due to the decreased viscosity compared to polymers. In addition, Tan et al [19] studied the impact properties of silica particles at concentrations of 20, 40, $50 \mathrm{wt} \%$ suspended in water due to water's spontaneous increased ability of impregnation, however, the rheological studies were not shown in the study. The viscosities of STF composed of 30 and $40 \mathrm{wt} \%$ fumed silica dispersed in PEG vs. shear rates are presented in figure 3. Shear thickening behaviour is attained by dispersing the fumed silica in PEG. The viscosity values are diminished when the shear rate is increased upto $\sim 9 \mathrm{~s}^{-1}$, which can be attributed to the shear thinning. Particle rearrangement, i.e., particle movement towards the direction of shear gradient rather than the flow direction led to decrease in Brownian force's contribution to the shear stress [2]. Therefore, shear thinning was observed since the shear viscosity equals to the ratio of shear stress to shear rate $[2,17]$. Shear thickening is detected after that point (with an increase in shear rate). Because, hydrodynamic forces and shear rate are directly proportional [2,17].

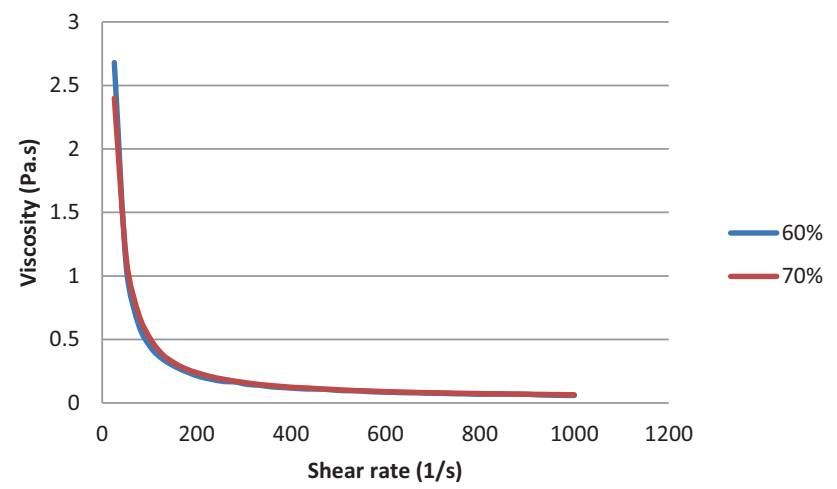

Figure 2. A plot of the rheological behaviour of the aqueous suspension of $\mathrm{SiO}_{2}$ particles at amounts of 60 and $70 \mathrm{wt} \%$.
So, hydrodynamic forces overwhelm by increasing shear rate. Furthermore, power law model was applied to the shear thickening portion of figure 3 . Because, it was stated that the model can represent the fluid where viscosity is increased by increasing shear rate and it fails at low shear rates $[4,6]$. Exponential trend lines of figure 3 were obtained and corresponding equations were determined as $\eta=190.95 \dot{\gamma}^{0.1413}$ and $\eta=188.07 \dot{\gamma}^{0.1168}$ for 30 and $40 \mathrm{wt} \%$ fumed silica, respectively. Therefore, average $m$ and $n$ values are calculated as 189.51 and 1.1291, which are $>1$ that represents shear thickening behaviour. No significant difference is observed between STFs at amounts of 30 and $40 \mathrm{wt} \%$ silica, but only slight increase is detected at the maximum viscosity value of those with $40 \mathrm{wt} \%$ silica.

\subsection{Impact test}

Neat and colloidal $\mathrm{SiO}_{2}$ in water-impregnated Kevlar samples with 1-5 numbers (table 1) were subjected to

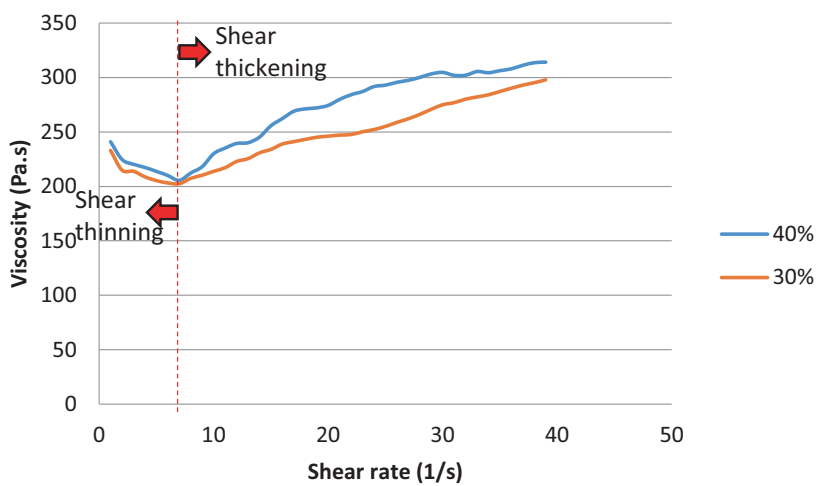

Figure 3. A plot of the rheological behaviour of fumed $\mathrm{SiO}_{2}$ particles at amounts of 30 and $40 \mathrm{wt} \%$.

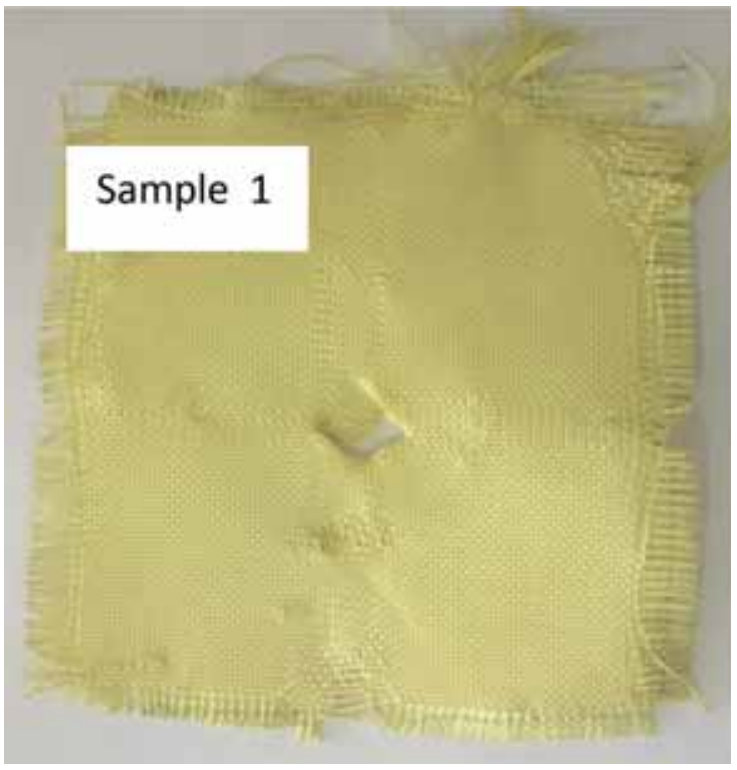

Figure 4. Neat Kevlar impact test with drop height of $0.2 \mathrm{~m}$. 


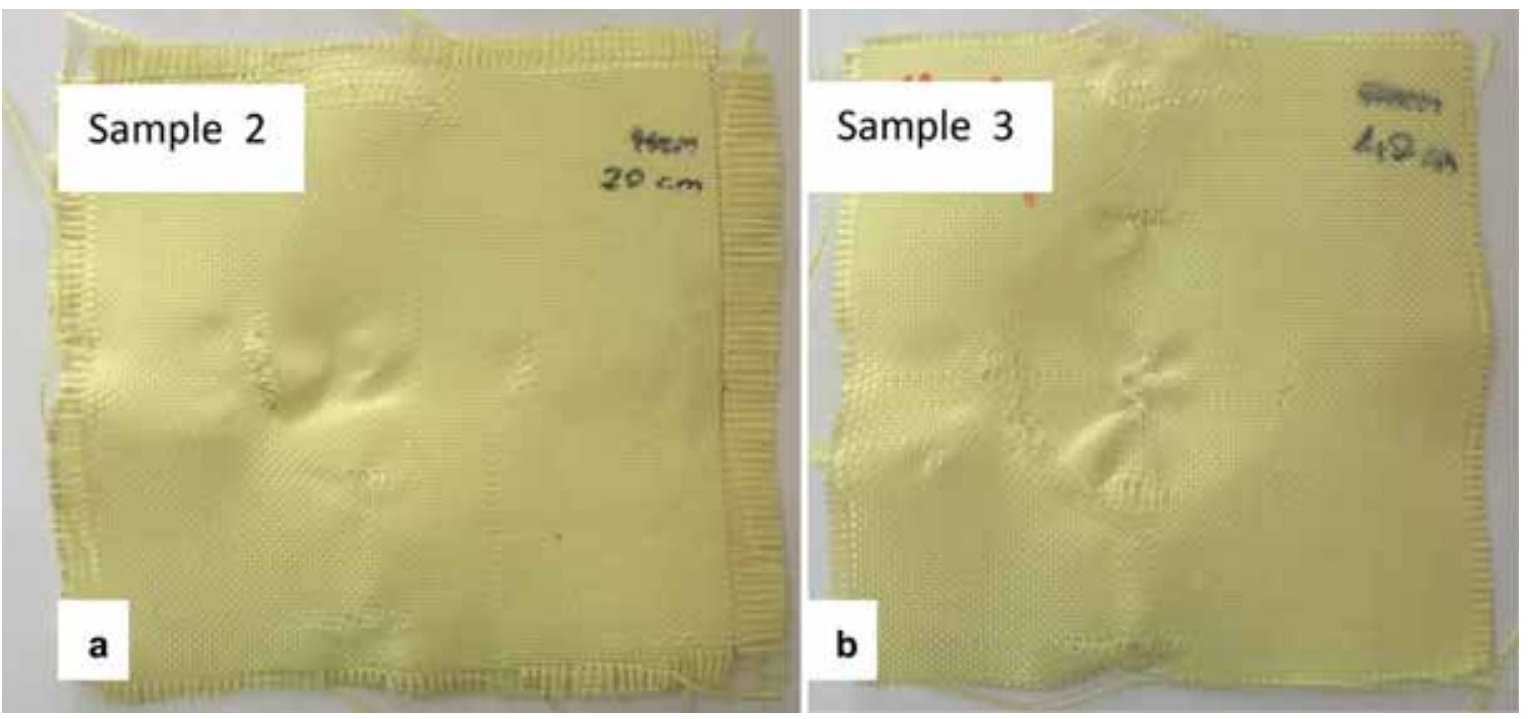

Figure 5. $40 \mathrm{wt} \% \mathrm{SiO}_{2}$ dispersed in water is impregnated on Kevlar and subjected to impact test with drop height of (a) 0.2 and (b) $0.48 \mathrm{~m}$.
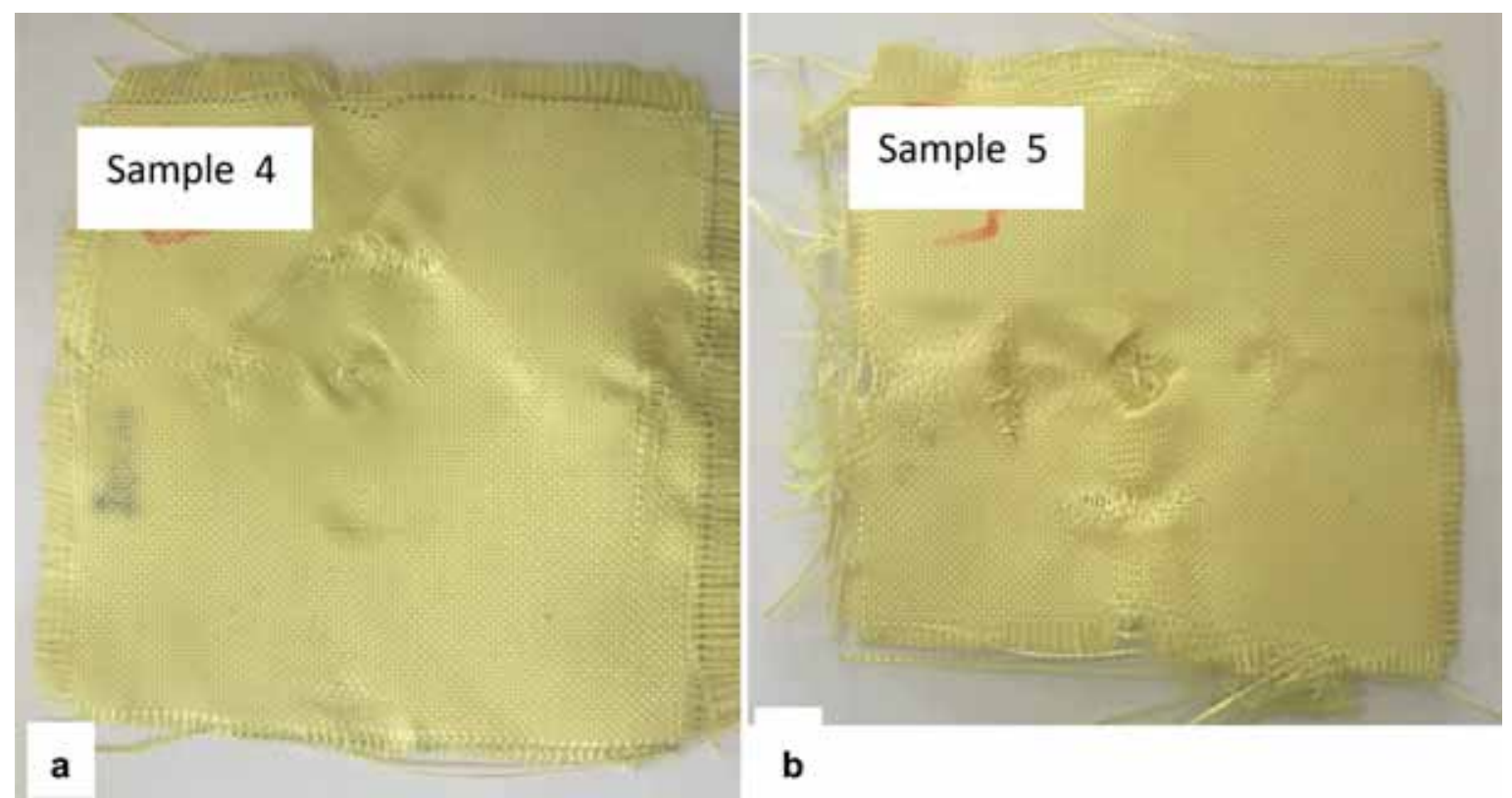

Figure 6. $70 \mathrm{wt} \% \mathrm{SiO}_{2}$ dispersed in water is impregnated on Kevlar and subjected to impact test with drop height of (a) 0.2 and (b) $0.48 \mathrm{~m}$.

impact test. Figure 4 demonstrates the penetration holes showing the effect of impact. Impregnation of dispersed $40 \mathrm{wt} \% \mathrm{SiO}_{2}$ in water caused broadening in the deformation area and less damage is shown in figure 5. The sample was not penetrated even if the drop height was increased from 0.2 to $0.48 \mathrm{~m}$ in the case of impregnation (figure 5). This improvement is not a result of shear thickening effect, since $\mathrm{SiO}_{2}$ in water-impregnated Kevlar samples did not show shear thickening behaviour (figure 2), but it is due to the filling of free volume within the fabric. Comparing figures 5 and 6 , displayed the effect wt $\%$ of $\mathrm{SiO}_{2}$ from 40 to $70 \%$. In addition, the lower silica concentration led to decreased displacement according to figure 7 , which is supported by figures $5 \mathrm{a}$ and $6 \mathrm{a}$. The result isconformed with the study of Tan et al, which claimed that the ballistic limit is increased by colloidal $\mathrm{SiO}_{2}$ in water 
treatment to Kevlar fabrics and increasing $\mathrm{SiO}_{2}$ wt\% from 40 to 50 led to slight decrease in the ballistic performance of the samples [19].

It is known that ply systems are preferred to improve the ballistic performance of impregnated Kevlar fabrics at the expense of increase in weight [19]. Ply systems with STFs composed of dispersed $40 \mathrm{wt} \%$ silica in PEG were studied to develop the performance. Figures 8 and 9 show the insignificant bulging difference between the 3- and 5-layers of STF-treated Kevlars mentioned as samples 6 and 7 in table 1 after impact test, respectively. Slight improvement was detected in the displacement value of 5-layers system after the weight drop according to figure 10 .

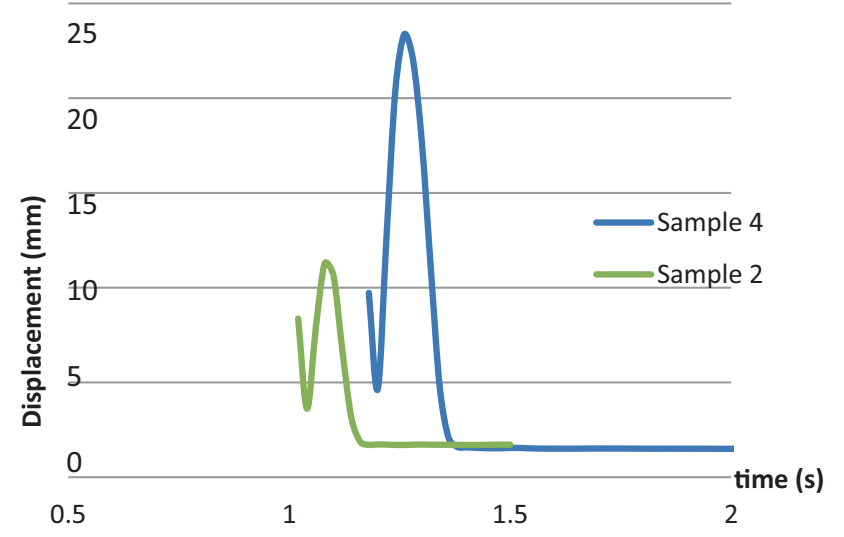

Figure 7. Displacement $v$ s. time graphs of samples 2 and 4 after drop test in table 1 .

\subsection{Flexibility test}

The flexibilities of neat Kevlar, 30 and $40 \mathrm{wt} \%$ fumed silica dispersed in PEG are shown in figure 11. It can be concluded that the least flexibility belongs to neat Kevlar and the maximum flexibility is achieved by the impregnation of $40 \mathrm{wt} \%$ fumed silica dispersed in PEG on Kevlar.

\subsection{Yarn pull-out test}

The results of single yarn pull-out tests for STF and neat Kevlar specimens are displayed in figure 12, which is a typical steady state pull-out stage of the yarn pull-out figures [43]. The reason for not observing the maximum peak load may be due to the minimum surface roughness values of the yarns. It is claimed that the peak pull-out force depends on the yarn-yarn static coefficient, which depends on the surface topology [43]. The oscillation seen in figure 12 may stem from the frictional force created by the transverse yarns related with the kinetic friction coefficient [43]. Decrease in the force was detected, once the yarn passed the transverse yarn, which is in contact with, and increase was observed when it encounters another transverse yarn. Moreover, STF-treated specimens exhibited larger loads (figure 12), which is consistent with the claim of PEG-impregnated Kevlar fabrics displayed higher peak loads and load amplitudes as compared to neat specimens [43,44]. Because, the yarns are no longer in contact with each other once they are impregnated due to the adherence of the solvent [43]. In addition, the force required to pull the yarn out in STF specimens was increased by increasing the pulling rate that triggers the shear thickening behaviour.
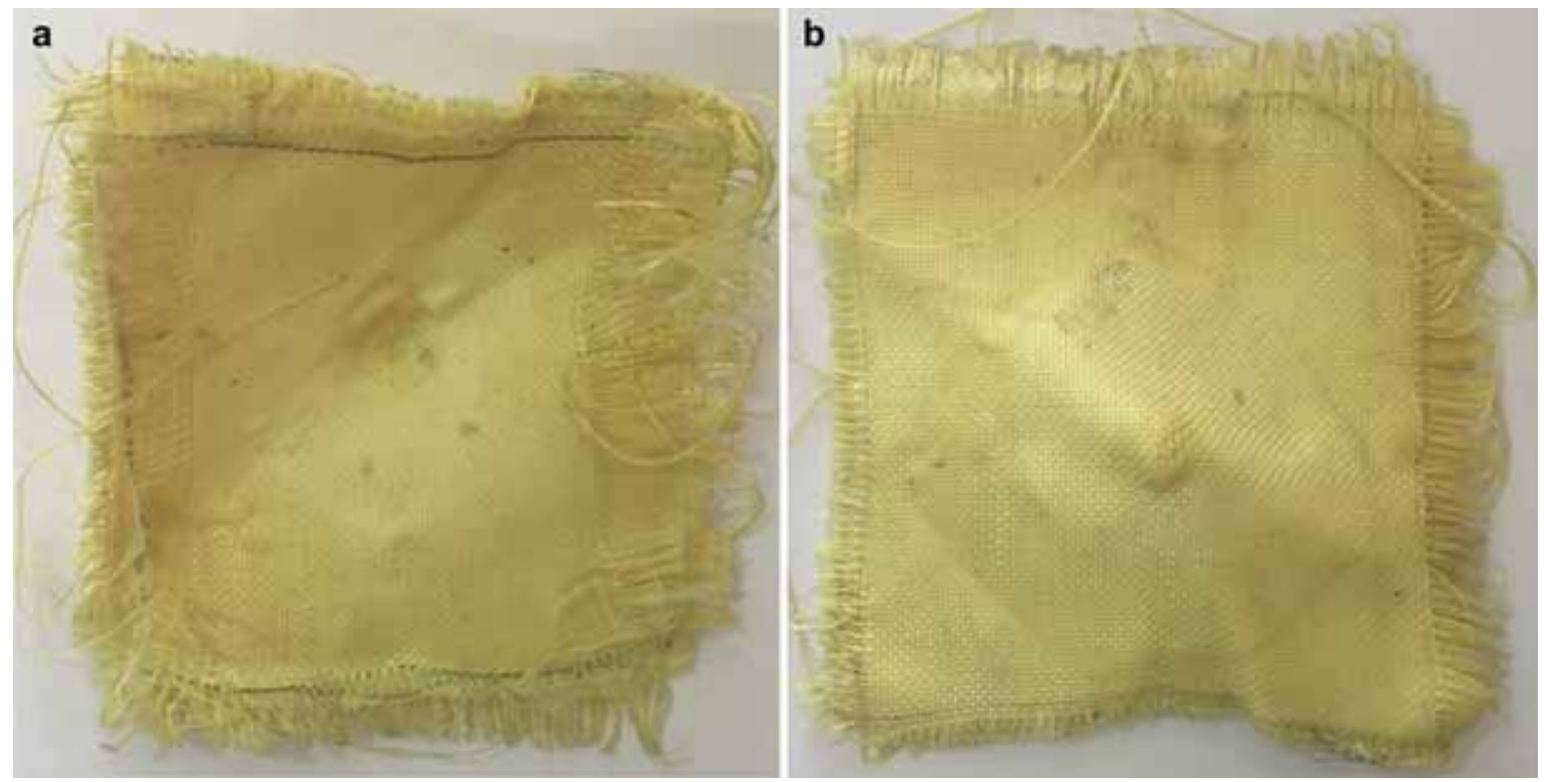

Figure 8. 3 Ply systems of $40 \mathrm{wt} \% \mathrm{SiO}_{2}$ dispersed in PEG impregnated Kevlar subjected to impact test with drop height of $1.77 \mathrm{~m}$. (a) Front view and (b) back view. 

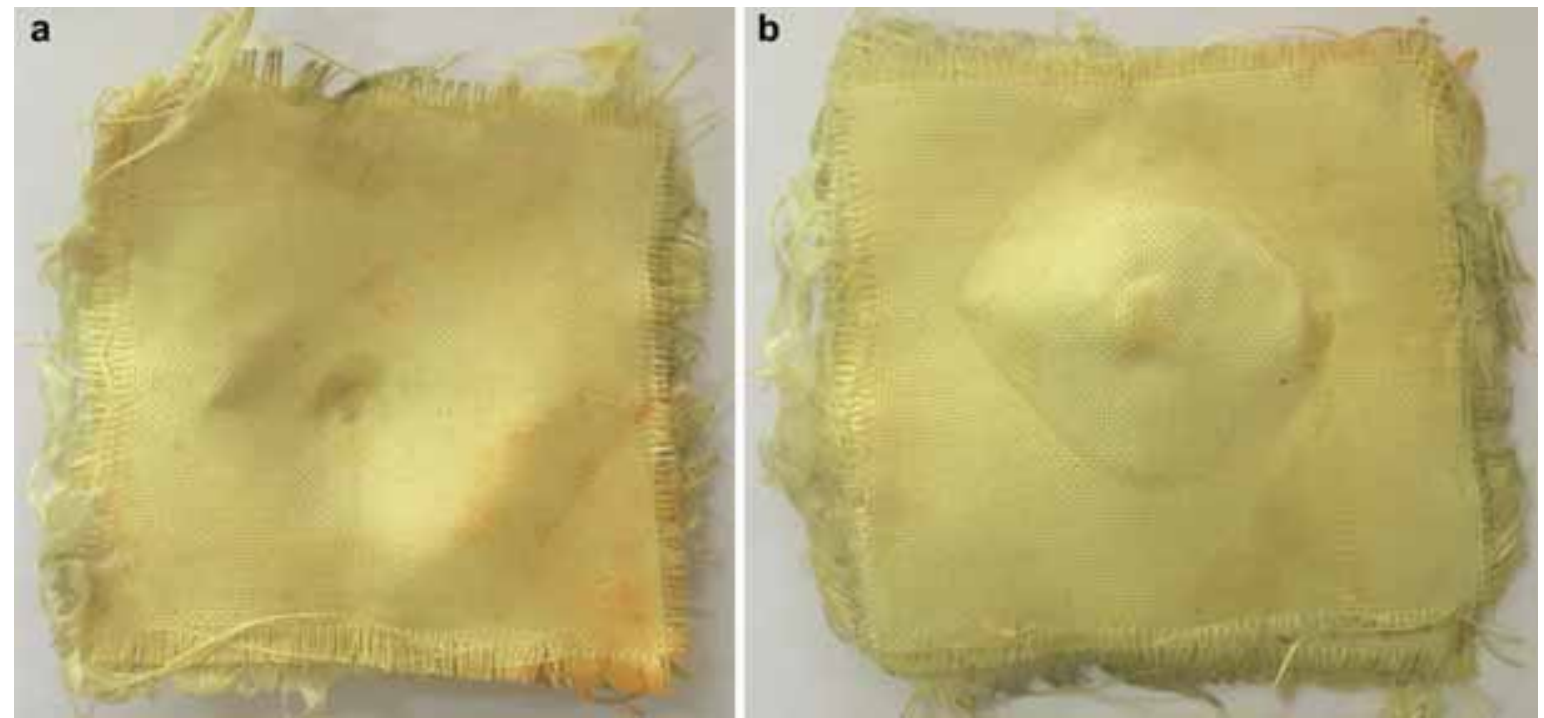

Figure 9. 5 Ply systems of $40 \mathrm{wt} \% \mathrm{SiO}_{2}$ dispersed in PEG impregnated Kevlar subjected to impact test with drop height of $1.77 \mathrm{~m}$. (a) Front view and (b) back view.
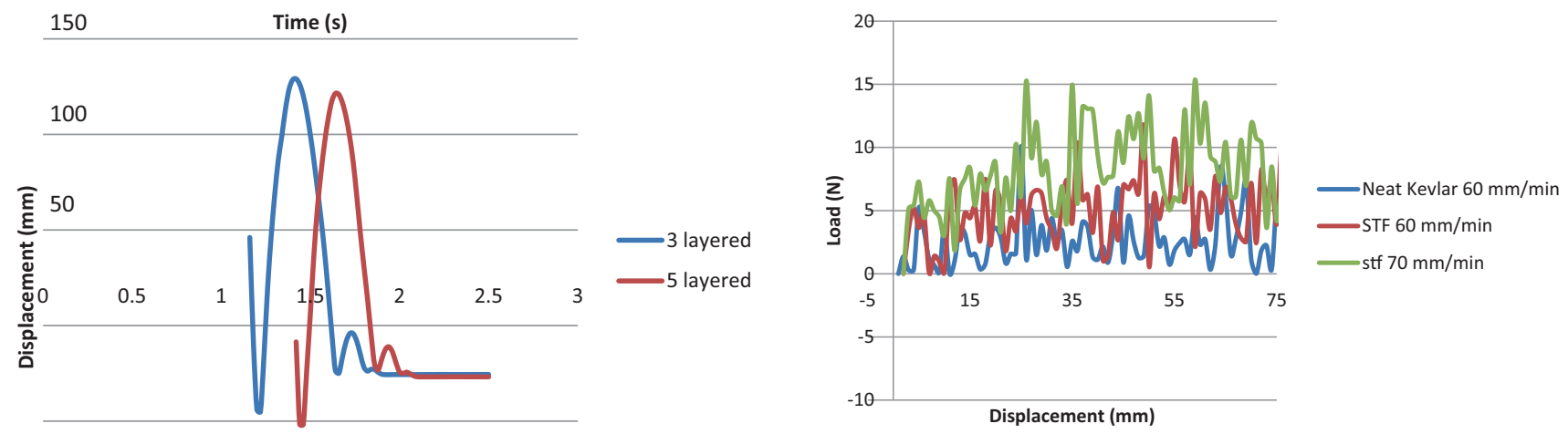

Figure 12. Pulling force-displacement curves for STF-treated and neat Kevlar samples at pull-out speeds of 60,70 and $60 \mathrm{~mm} \mathrm{~min}^{-1}$,

Figure 10. $40 \mathrm{wt} \% \mathrm{SiO}_{2}$ dispersed in $\mathrm{PEG}$ is impregnated on 3 and 5 ply Kevlar and subjected to impact test with drop height of $1.77 \mathrm{~m}$.

3

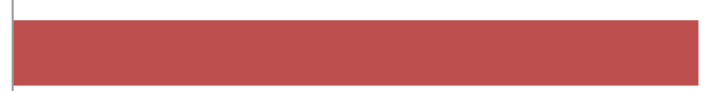

2

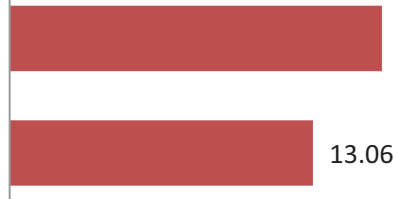

15.66

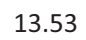

\subsection{6}

14

15

16
11

13

Displacement (mm)

Figure 11. Flexibility test results of (1) Kevlar, (2) $30 \%$ silica + PEG and (3) 40\% silica + PEG.

\section{Conclusion}

Shear thickening behaviour was not observed when silica at amounts of 60 and $70 \mathrm{wt} \%$ dispersed in water. However, impregnated sample by dispersed $40 \mathrm{wt} \% \mathrm{SiO}_{2}$ in water were not penetrated when subjected to impact test at both drop heights of 0.2 and $0.48 \mathrm{~m}$, where fibre breakage and hole formation were observed in neat Kevlar even at the drop height of $0.2 \mathrm{~m}$. In addition, the displacement occurred in the fabrics was increased when the silica concentration was increased from 40 to $70 \%$.

Fumed silica dispersed in PEG led to obtain increased viscosity with an increase in the shear rate. In addition, the maximum point of viscosity showed a minor increase by rising the wt $\%$ of silica from 30 to 40 . The flexibility of STF was higher than neat Kevlar. Moreover, increasing fumed silica amount in PEG of STF from 30 to $40 \mathrm{wt} \%$ 
led to an increment in the flexibility. The maximum force attained during single yarn pull-out test was increased in STFtreated Kevlar (40\% fumed $\mathrm{SiO}_{2}$ in PEG) as compared to neat Kevlar. Furthermore, increasing the pull-out speed from 60 to $70 \mathrm{~mm} \mathrm{~min}^{-1}$ during the single yarn pull-out tests of STFtreated Kevlar specimens led to increment in the maximum force.

\section{References}

[1] Srivastava A, Majumdar A and Butola B S 2011 Mater. Sci. Eng. A 529224

[2] Mewis J and Wagner N J 2012 Colloidal suspension rheology (Cambridge: Cambridge University Press)

[3] Petel O E, Ouellet S, Loiseau J, Frost D L and Higgins A J 2015 Int. J. Impact Eng. 8583

[4] Macosko C W 1994 Rheology: principles, measurements and applications (Canada: Wiley VCH)

[5] Marcotte M, Taherian Hoshahili A R and Ramaswamy H S 2001 Food Res. Int. 34695

[6] Galindo-Rosales F J, Rubio-Hernández F J and Sevilla A 2011 J. Nonnewton. Fluid Mech. 166321

[7] Raghavan S and Khan S 1997 J. Colloid Interface Sci. 18557

[8] Barnes H A 1989 J. Rheol. 33329

[9] Hasanzadeh M and Mottaghitalab V 2014 ASM Int. 231182

[10] Rangari V K, Hassan T A, Mahfuz H and Jeelani S 2006 NSTI Nanotech. Tech. Proceeding 2637

[11] Zhang X Z, Li W H and Gong X L 2008 Smart Mater. Struct. 1735027

[12] Yu K, Cao H, Qian K, Sha X and Chen Y 2012 J. Nanopart. Res. 14747

[13] Park J L, Yoon B I, Paik J G and Kang T J 2012 Text. Res. J. 86527

[14] Sun L L, Xiong D S and Xu C Y 2013 J. Appl. Polym. Sci. 129 1922

[15] Boersma W H, Laven J and Stein H N 1995 J. Rheol. 39841

[16] Boersma W H, Laven J and Stein H N 1990 AIChE J. 36321

[17] Liu X Q, Bao R Y, Wu X J, Yang W, Xie B H and Yang M B 2015 RSC Adv. 518367

[18] Foss D R and Brady J F 2000 J. Fluid Mech. 407167

[19] Tan V B C, Tay T E and Teo W K 2005 Int. J. Solids Struct. 421561
[20] Petel O E, Ouellet S, Loiseau J, Marr B J, Frost D L and Petel O E 2013 Appl. Phys. Lett. 10264103

[21] Lee Y S, Wetzel E D and Wagner N J 2003 J. Mater. Sci. 38 2825

[22] Yu K, Cao H, Qian K and Jiang L 2012 Fibres Text. 95126

[23] Hassan T A, Rangari V K and Jeelani S 2010 Mater. Sci. Eng. A 5272892

[24] Park Y, Kim Y, Baluch A H, Kim C, Çerkez İ and Horizons N 2014 Int. J. Impact Eng. 671561

[25] Kordani N and Vanini A S 2014 Sci. Eng. Compos. Mater. 21 421

[26] Warren J, Offenberger S, Toghiani H, Pittman C U, Lacy T E and Kundu S 2015 ACS Appl. Mater. Interfaces 718650

[27] Minagawa N and White J L 1976 J. Appl. Polym. Sci. 20 501

[28] Kordani N and Vanini A S 2014 J. Mech. Sci. Technol. 28 663

[29] Wetzel E D, Lee Y S, Egres R G, Kirkwood K M, Kirkwood J E and Wagner N J 2004 AIP Conf. Proc. 712288

[30] Kang T J, Kim C Y and Hong K H 2012 J. Appl. Polym. Sci. 1241534

[31] Mewis J and Biebaut G 2001 J. Rheol. 45799

[32] Kim S Y and Zukoski C F 2011 Langmuir 2710455

[33] Taylor P, Chamberlain E K, Rao M A and Cohen C 1998 Int. J. Food Prop. 263

[34] Wiśniewska M, Szewczuk-Karpisz K and Ostolska I 2013 Fluid Phase Equilib. 36010

[35] Decker M J, Halbach C J, Nam C H, Wagner N J and Wetzel E D 2007 Compos. Sci. Technol. 67565

[36] Lee B W and Kim C G 2012 Adv. Compos. Mater. 21177

[37] Park Y, Kim Y, Baluch A H and Kim C G 2015 Compos. Struct. 125520

[38] Lu Z, Jing X, Sun B and Gu B 2013 Compos. Sci. Technol. 88 184

[39] Dong Z and Sun C T 2009 Compos. Part A: Appl. Sci. Manuf. 401863

[40] Nilakantan G and Gillespie J W 2013 Compos. Struct. 101215

[41] Lee B W, Kim I J and Kim C G 2009 J. Compos. Mater. 43 2679

[42] Bilisik K 2012 J. Ind. Text. 41201

[43] Grujicic M, Sellappan V, Omar M A, Seyr N, Obieglo A and Erdmann M 2008 J. Mater. Process. Technol. 197363

[44] Kalman D P, Merrill R L, Wagner N J and Wetzel E D 2009 ACS Appl. Mater. Interfaces 12602 